\title{
The global state of research and trends in osteomyelitis from 2010 to 2019: a 10-year bibliometric analysis
}

\author{
Peisheng Chen ${ }^{1 \#}$, Xiaofeng Lin ${ }^{2 \#}$, Bin Chen ${ }^{1}$, Ke Zheng ${ }^{1}$, Chaohui Lin $^{1}$, Bin Yu ${ }^{3}$, Fengfei Lin ${ }^{1,4}$ \\ ${ }^{1}$ Department of Orthopaedics, Fuzhou Second Hospital Affiliated to Xiamen University, Teaching Hospital of Fujian Medical University, Fuzhou, \\ China; ${ }^{2}$ Department of Endocrinology, Fuzhou Second Hospital Affiliated to Xiamen University, Teaching Hospital of Fujian Medical University, \\ Fuzhou, China; ${ }^{3}$ Department of Orthopaedics and Traumatology, Nanfang Hospital, Southern Medical University, Guangzhou, China; ${ }^{4}$ Trauma \\ Medical Center of Fuzhou City, Fuzhou Second Hospital Affiliated to Xiamen University, Fuzhou, China \\ Contributions: (I) Conception and design: P Chen, F Lin; (II) Administrative support: F Lin; (III) Provision of study materials or patients: P Chen; \\ (IV) Collection and assembly of data: X Lin, B Chen; (V) Data analysis and interpretation: P Chen, X Lin, B Chen, K Zheng, C Lin, B Yu; (VI) \\ Manuscript writing: All authors; (VII) Final approval of manuscript: All authors. \\ \#These authors contributed equally to this work. \\ Correspondence to: Peisheng Chen. Department of Orthopaedics, Fuzhou Second Hospital Affiliated to Xiamen University, Teaching Hospital of \\ Fujian Medical University, Fuzhou 350007, China. Email: wsbzcps@163.com; Fengfei Lin. Department of Orthopaedics, Fuzhou Second Hospital \\ Affiliated to Xiamen University, Teaching Hospital of Fujian Medical University, Fuzhou 350007, China. Email: 596558644@qq.com.
}

Background Osteomyelitis is a difficult problem for orthopedic surgeons due to its great harm and complicated treatment. In this study, we aim to make a bibliometric analysis of the literature related to osteomyelitis and explore the research status, hotspots and frontiers in this field in recent 10 years.

Methods: Literature relating to osteomyelitis from 2010 to 2019 was retrieved from the database of Science Citation Index Expanded (SCIE) of Web of Science. CiteSpace was used to analyze country/institution, authors/cited authors, cited journals, cited references, and keywords. An analysis of counts and centrality was used to reveal publication outputs, countries/institutions, core journals, active authors, hot topics, and frontiers.

Results: A total of 6,421 valid literatures were retrieved. The most productive country and institution were the United States and Shanghai Jiao Tong University, respectively. Researchers and institutions from the United States, Germany, England, and France were the core research forces. There was a broad and close cooperation worldwide. Lipsky BA [24] was the most productive first author, and Lew DP [487] was the most frequently cited author. Lipsky et al.'s [2012] article (co-citation counts, 146) was the most representative and symbolic reference. Fournal of Foot Ankle Surgery [111] was the most productive journal. Clin Infect Dis [2,275] was the most frequently co-cited journal. Staphylococcus aureus infection and the diagnosis, treatment and management strategy of osteomyelitis were the hot spots. Epidemiology, diabetic foot, treatment, especially antibiotics, biofilm and in vitro research were research frontiers.

Conclusions: This study reveals the current research status and hot spots in the field of osteomyelitis in recent 10 years, which may help researchers to identify further potential perspectives on collaborators, research frontiers, and hot topics.

Keywords: Osteomyelitis; bibliometric analysis; hotspots; medical information sciences

Submitted Oct 04, 2020. Accepted for publication Dec 31, 2020.

doi: 10.21037/apm-20-1978

View this article at: http://dx.doi.org/10.21037/apm-20-1978 


\section{Introduction}

Osteomyelitis is usually defined as an inflammatory disease of bone caused by infectious pathogens (1). The major sources of infection are haematogenous spread, tracking from adjacent foci of infection, and direct inoculation from trauma or surgery. Hematogenous osteomyelitis and septic arthritis are mainly seen in children (2). The long bone metaphysis (in the growth stage) area of children has a rich and slow blood flow, and even minor trauma is extremely vulnerable (3). In children younger than 5 years of age, more than half of hematogenous osteomyelitis cases occur, especially acute hematogenous osteomyelitis. Staphylococcus aureus is the most common pathogen of musculoskeletal infections (1). Over the past few decades, the pattern of pathogenic microorganisms has been changing, with more resistant strains emerging, and the main pathogenic bacteria are slowly changing from gram-positive bacteria to gramnegative bacteria.

Diagnosis of osteomyelitis is often difficult, and treatment is relatively tricky. Once the infection cannot be effectively controlled in time, it may lead to serious consequences such as reoperation, long-term antibiotic treatment, high medical costs, inability to work and live normally, loss of limb function or even amputation (4). Therefore, for this destructive bone infection, there is an urgent need to further improve the understanding of it, and take positive and decisive measures, targeted planning actions, and strive for early detection, early diagnosis and early treatment.

Bibliometrics is a mature research method in information science, and it has been proven to be an effective tool for studying the state of subjects and reflecting its development objectively (5). The knowledge graph drawing tool represented by CiteSpace, through the organic combination of applied mathematics, statistics, bibliometrics, information science and other multi-disciplinary methods, visually shows the development process and research hotspots of a research field $(6,7)$. At present, this method has been widely used in the hot spot analysis of various diseases, and provides a reference basis for further research on prevention and treatment of diseases (8-11). However, there have been few bibliometric studies on osteomyelitis. In view of this, we provide an integrated and in-depth analysis of the content and external features of the research on osteomyelitis and summarize past research and predict future research hotspots.

\section{Methods}

\section{Data sources and search strategies}

Bibliometric analysis was performed using the Science Citation Index-Expanded (SCIE) of Web of Science database. We comprehensively searched Web of Science database to find relevant data from 2010 to 2019 and only included original articles and reviews. The search strategy was presented as follows: $\mathrm{TS}=$ (osteomyelitis) AND Language $=$ English. To avoid bias incurred by frequent database renewal, all literature retrieval and data downloads were completed in a single day, May 27, 2020.

\section{Data collection}

Two researchers (XFL and BC) independently verified the data entry and collection. Differences between the two researchers' verifications were discussed between these researchers to reach consensus (12). Finally, the data were downloaded from the Web of Science database and imported into software CiteSpace V5.6.R5 SE, 64bit (Drexel University, Philadelphia, PA, USA) for bibliometric analysis.

\section{Statistical analysis}

Bibliometrics is a statistical method that can quantitatively analyze research papers related to a specific topic through mathematical methods. We tried to describe the characteristics of all publications. CiteSpace is an effective bibliometric analysis tool for analyzing the networks distribution characteristics of various publication, and can obtain clustering keywords to predict the research frontiers and emerging trends in this area. In terms of parameter setting, the time slicing was set to "From 2010 JAN TO 2019 DEC, 1 year per slice". The term sources were selected as "Title", "Abstract", "Author Keywords (DE)" and "Keywords Plus (ID)", and the node types were selected as "Author", "Institution", "Country", "Reference", "Cited Author", "Cited Journal", and "keyword". Links strength was selected as "cosine (cosine function)". Selection criteria was selected as "TOP N", selected top 20 levels of most cited or occurred items from each slice. Pruning sliced networks was selected for the pruning process. Through the co-citation analysis, the cited frequency and centrality of the nodes were calculated, and then the key data in the development of this research field were found.

The visual map was drawn step by step according to the 


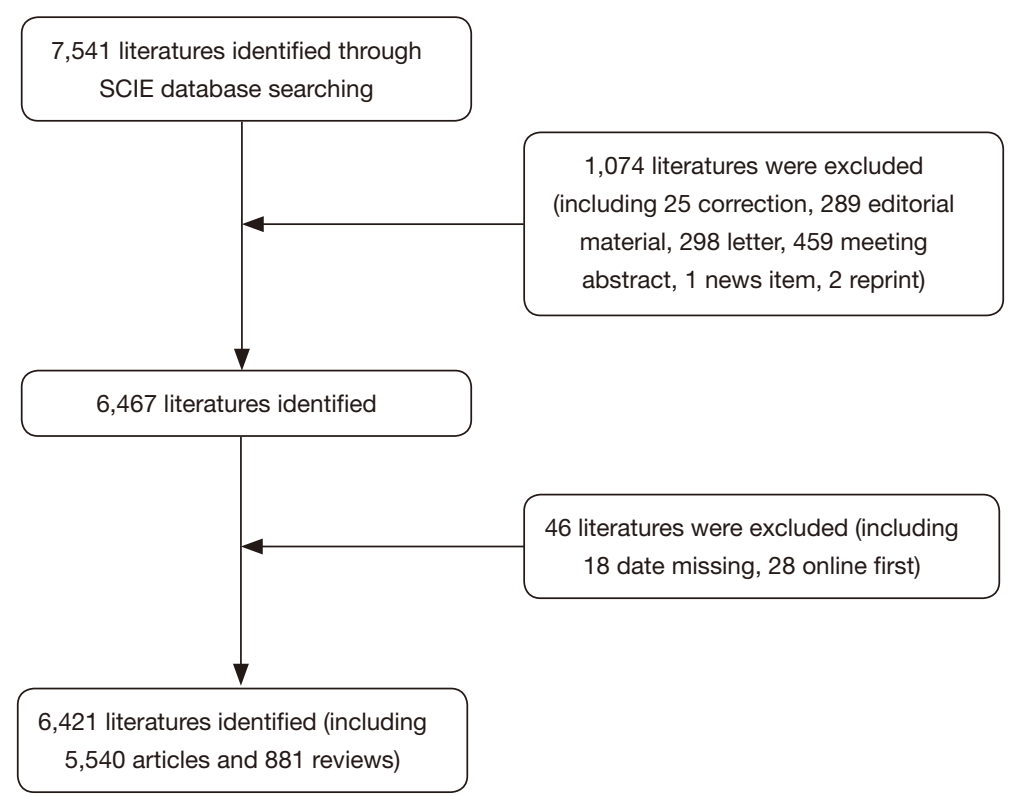

Figure 1 Flow chart of literature filtering included in this study. SCIE, Science Citation Index-Expanded.

setting parameters by CiteSpace. In the map, the nodes represent the analyzed objects, and the more frequency, the larger the nodes. The color and thickness in the inner circle of the node indicated the occurrence or cited frequency of different time periods. The color of the nodes corresponded to the time of its first co-occurrence or co-citation, and the change from cold tone to warm tone indicated the change of time from early to recent. The edge between nodes and their thickness represents the relationship and strength of co-occurrence or co-citation, respectively. The nodes with centrality $\geq 0.1$ were marked with purple circle. To survey the hotspots of osteomyelitis study, we carried out a series of keyword analysis of the incorporated publications, including keywords co-occurrence, keywords clustering and keywords with the strongest citation bursts analysis.

\section{Results}

\section{Evaluation of global publications}

A total of 6,421 publications $(5,540$ articles and 881 reviews) were identified (Figure 1). In general, the numbers of osteomyelitis-related publications per year indicated a steadily increasing trend over the past 10 years (from 581 in 2010 to 761 in 2019, Figure 2).

\section{The contributions of countries/regions and institutions to global publications}

Most of the countries/regions or institutions with high production located in Europe and the United States. The network map of countries/regions included 29 nodes and 206 edges, as shown in Figure 3A. The United States [2,129] was the largest contributor to osteomyelitis research, followed by China [496], Germany [416], England [408], and France [359]. Centrality is a major indicators to determine the importance of nodes in the network and a higher centrality means that the node is more important in this network. The nodes with centrality $\geq 0.1$ are marked with purple circle. Therefore, the results showed that the United States had more impact than any other country (centrality $=0.27$ ), and then Japan [0.19], and Germany [0.11] (Table 1).

In terms of research institutions, the number of nodes was 121 and the number of edges was 175 in the network map (Figure 3B). The top 5 institution included Shanghai Jiaotong University \{China, [67]\}, Mayo Clinic \{United States, [66]\}, University of Washington \{United States, [54]\}, University of California, San Francisco \{United States, [39]\}, University of Oxford \{United Kingdom, [37]\} (Table 1). Centrality analysis showed that the University 


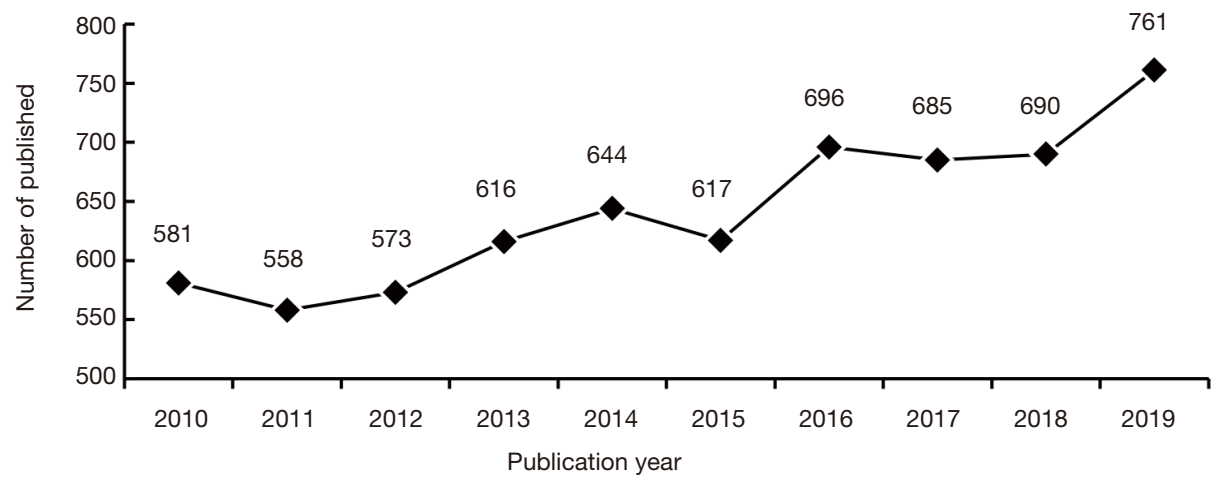

Figure 2 Output of related literature. The number of annual publications in osteomyelitis research from 2010 to 2019.

A

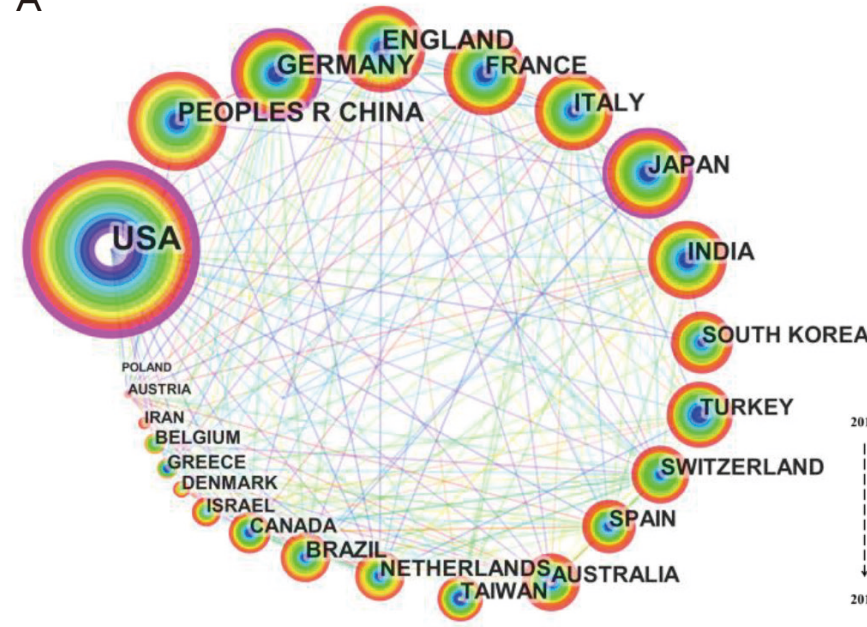

B

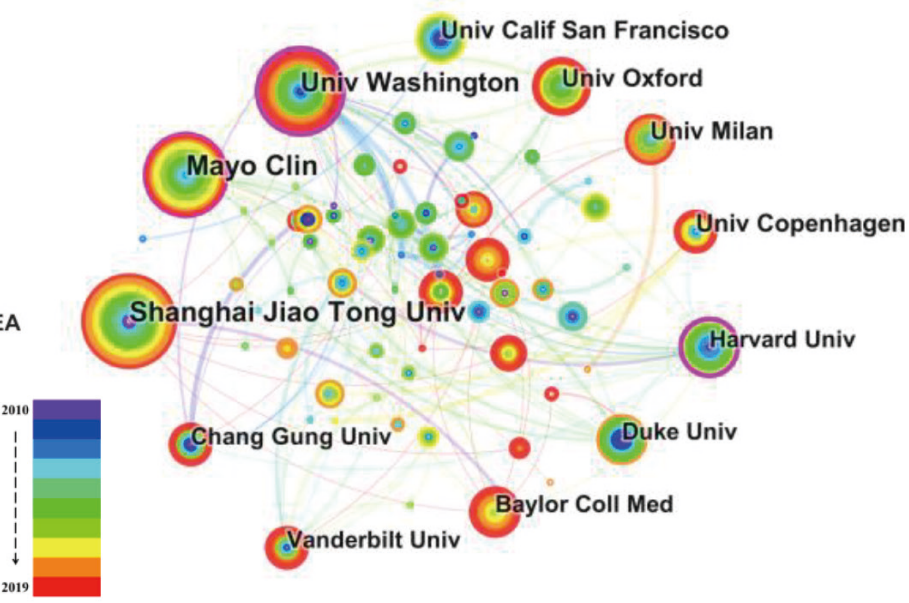

Figure 3 The distribution of countries/regions and institutions. The network map of countries/regions involved in osteomyelitis research (A) and cooperation between institutions (B). In the map, the nodes represent the analyzed objects, and the more frequency, the larger the nodes. The color and thickness in the inner circle of the node indicated the occurrence or cited frequency of different time periods. The edge between nodes and their thickness represents the relationship and strength of co-occurrence or co-citation, respectively. The nodes with centrality $\geq 0.1$ were marked with purple circle. Univ, university.

of Washington had more impact than any other research institutions (centrality $=0.30$ ), and then Harvard University [0.23], and Mayo Clinic [0.15] (Table 1).

\section{Fournals publishing researches on osteomyelitis}

Recently, 1,436 journals have appeared in the field of osteomyelitis research. The top 20 most popular journals published 984 of all 6,421 pieces $(15.32 \%)$ of literature on osteomyelitis in our study (Figure $4 A$ ), which means that the publications were relatively scattered. Of these, the top 5 journals were fournal of Foot Ankle Surgery [111], Pediatric
Infectious Disease Fournal [84], Injury International Fournal of the Care of the Injured [79], PLoS One [72], BMC Infectious Diseases [69], which accounted for more than $42.17 \%$ of all the top 20 journals. In the network map of co-cited journals, the number of nodes was 38 and the number of edges was 196 (Figure 4B). Of these, the top 5 journals were Clinical Infectious Diseases [2,275], The Fournal of Bone and foint Surgery-American Volume [1,957], Clinical Orthopaedics and Related Research [1,928], New England fournal Of Medicine $[1,616]$, and The Fournal of Bone and Foint SurgeryBritish Volume [1,465]. Miraculously, the centrality of the top 5 journals were all greater than 0.1 , indicating that they 
Table 1 The top 20 countries/regions and institutions contributing to publications in osteomyelitis research

\begin{tabular}{|c|c|c|c|c|c|c|c|c|}
\hline Rank & \multicolumn{4}{|c|}{ Countries/regions } & \multicolumn{4}{|c|}{ Institutions } \\
\hline 1 & 2,129 & 27 & 0.27 & USA & 67 & 8 & 0.07 & Shanghai Jiao Tong Univ \\
\hline 2 & 496 & 9 & 0 & China & 66 & 16 & 0.15 & Mayo Clin \\
\hline 3 & 416 & 22 & 0.11 & Germany & 54 & 26 & 0.3 & Univ Washington \\
\hline 5 & 359 & 21 & 0.1 & France & 37 & 5 & 0.01 & Univ Oxford \\
\hline 6 & 329 & 16 & 0.03 & Italy & 36 & 4 & 0.02 & Univ Milan \\
\hline 7 & 299 & 17 & 0.19 & Japan & 34 & 5 & 0.03 & Univ Copenhagen \\
\hline 8 & 249 & 18 & 0.08 & India & 33 & 19 & 0.23 & Harvard Univ \\
\hline 11 & 195 & 20 & 0.06 & Switzerland & 32 & 13 & 0.06 & Vanderbilt Univ \\
\hline 12 & 192 & 20 & 0.08 & Australia & 31 & 6 & 0.1 & Chang Gung Univ \\
\hline 13 & 192 & 17 & 0.03 & Spain & 30 & 9 & 0.05 & Univ Rochester \\
\hline 14 & 167 & 8 & 0.01 & Taiwan & 30 & 9 & 0.03 & Harvard Med Sch \\
\hline 15 & 162 & 19 & 0.06 & The Netherlands & 29 & 0 & 0 & Seoul Natl Univ \\
\hline 16 & 158 & 14 & 0.02 & Brazil & 27 & 6 & 0.01 & Univ Groningen \\
\hline 17 & 140 & 18 & 0.03 & Canada & 26 & 1 & 0 & Southern Med Univ \\
\hline 18 & 79 & 12 & 0 & Israel & 25 & 2 & 0.02 & China Med Univ \\
\hline
\end{tabular}

Univ, university.

have occupied a vital position in the field of osteomyelitis research, and can provide an important reference for osteomyelitis related research (Table 2). Specifically, The Fournal of Bone and Foint Surgery-American Volume (centrality $=0.35)$ ranked first in the top 5 co-cited journals, followed by Clinical Orthopaedics and Related Research [0.31], Clinical Infectious Diseases [0.27], New England Fournal of Medicine [0.19], and The Fournal of Bone and Foint Surgery-British Volume [0.15].

\section{The contributions of authors to osteomyelitis research}

We analyzed the information of cooperation between authors and co-cited authors, visualizing them in a network by CiteSpace (Figure 5). The number of nodes and edges in the network map of cooperation between authors was
464 and 763 respectively (Figure 5A). Of these, Benjamin A Lipsky, Professor of Medicine Emeritus at University of Washington, ranked first [24], followed by Javier AragónSánchez [21], Ilker Uçkay [19], Edward M. Schwarz [16], and Frederic Laurent [16]. These five scholars have made tremendous achievements and have become authorities in osteomyelitis research.

The network map of co-cited authors consisted of 64 nodes and 400 edges (Figure 5B). Lew DP (487 co-citations) ranked first in the top 5 co-cited authors, followed by Zimmerli W [373], Lipsky BA [335], Darouiche RO [246], and Cierny G [204] (Table 3). Lew DP had more impact in osteomyelitis field than any other authors (centrality $=0.50$ ), followed by Zimmerli W [0.29], Cierny G [0.12], and Darouiche RO [0.11]. The high centrality of these authors showed that they have become an influential core role in 
A

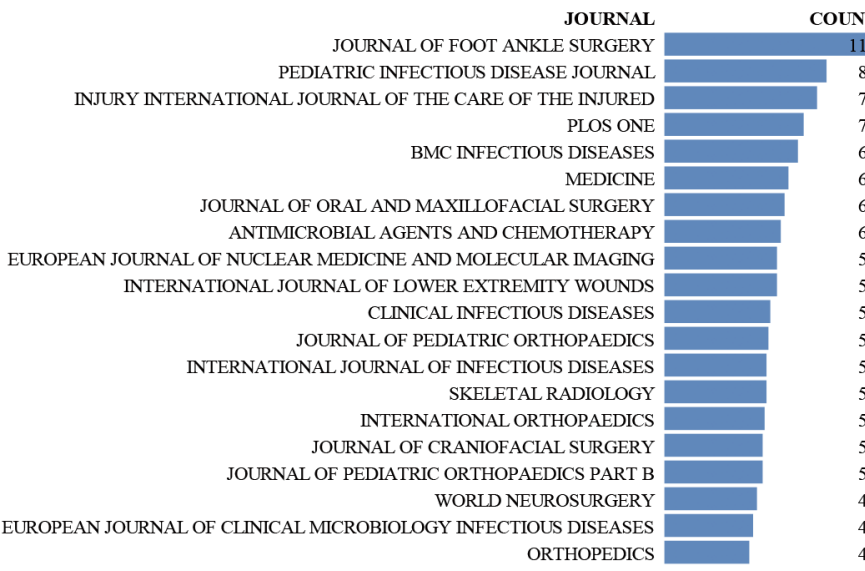

B

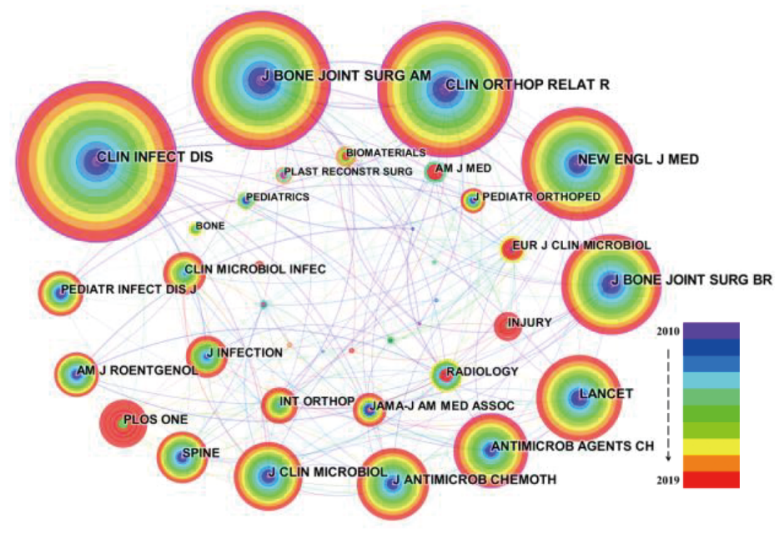

Figure 4 The distribution of journals publishing researches on osteomyelitis. The top 20 most active journals that published literatures in osteomyelitis research (sorted by count) (A), and the network map of co-cited journals (B). In the map, the nodes represent the analyzed objects, and the more frequency, the larger the nodes. The color and thickness in the inner circle of the node indicated the occurrence or cited frequency of different time periods. The edge between nodes and their thickness represents the relationship and strength of cooccurrence or co-citation, respectively. The nodes with centrality $\geq 0.1$ were marked with purple circle.

Table 2 The top 20 co-cited journals in the field of osteomyelitis research

\begin{tabular}{|c|c|c|c|c|}
\hline Rank & Article count & Degree & Centrality & Journal \\
\hline 2 & 1,957 & 22 & 0.35 & J Bone Joint Surg Am \\
\hline 3 & 1,928 & 22 & 0.31 & Clin Orthop Relat $R$ \\
\hline 4 & 1,616 & 20 & 0.19 & New Engl J Med \\
\hline 6 & 1,271 & 16 & 0.07 & Lancet \\
\hline 7 & 1,084 & 16 & 0.11 & Antimicrob Agents Ch \\
\hline 8 & 1,081 & 15 & 0.05 & $J$ Antimicrob Chemoth \\
\hline 9 & 998 & 13 & 0.03 & J Clin Microbiol \\
\hline 12 & 667 & 6 & 0.01 & Am J Roentgenol \\
\hline 13 & 662 & 7 & 0.01 & Pediatr Infect Dis $J$ \\
\hline 14 & 638 & 12 & 0.02 & Clin Microbiol Infec \\
\hline 15 & 631 & 13 & 0.04 & $J$ Infection \\
\hline 16 & 550 & 8 & 0.02 & Int Orthop \\
\hline 17 & 526 & 8 & 0 & JAMA-J Am Med Assoc \\
\hline 18 & 509 & 7 & 0.03 & Radiology \\
\hline 19 & 412 & 6 & 0 & Injury \\
\hline
\end{tabular}


A

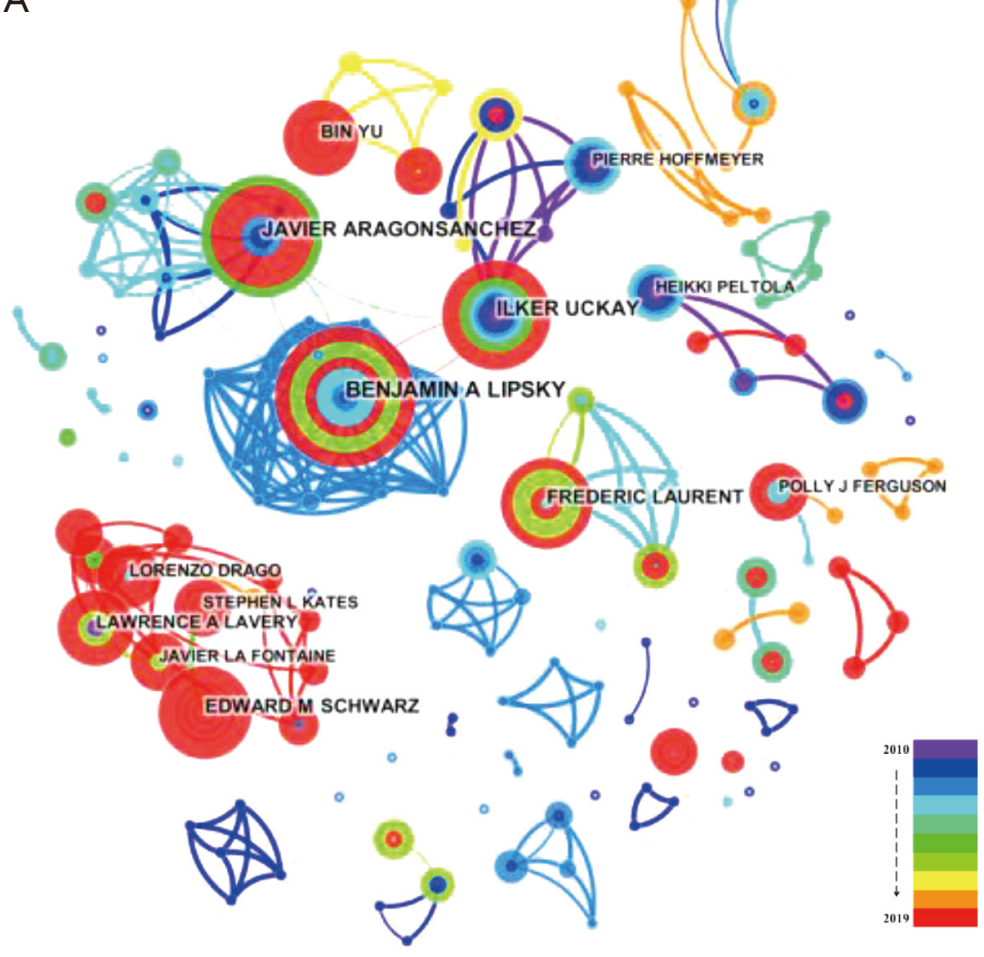

B

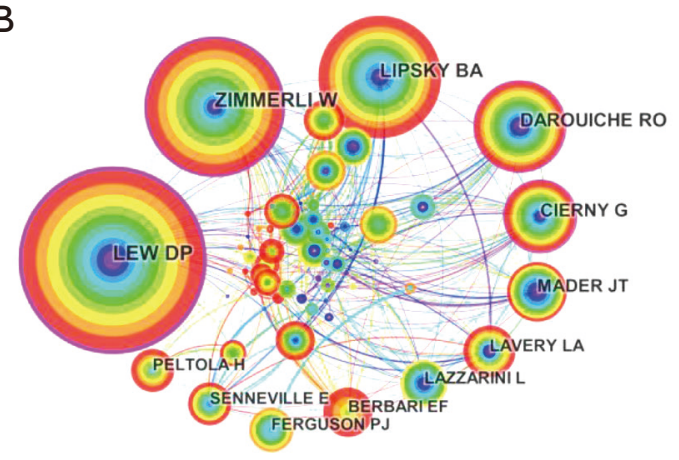

C

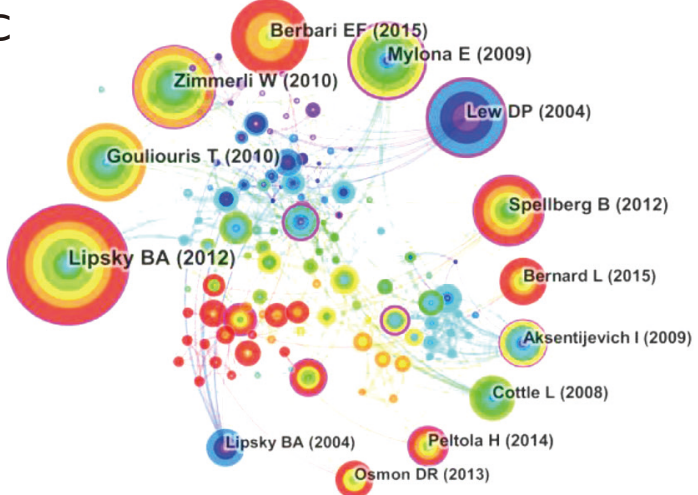

Figure 5 The distribution of authors engaged in osteomyelitis research. The network map of cooperation between authors (A). The network map of co-cited authors (B). The network map of co-occurrence analysis of authors and cited literatures (C). In the map, the nodes represent the analyzed objects, and the more frequency, the larger the nodes. The color and thickness in the inner circle of the node indicated the occurrence or cited frequency of different time periods. The edge between nodes and their thickness represents the relationship and strength of co-occurrence or co-citation, respectively. The nodes with centrality $\geq 0.1$ were marked with purple circle.

the field of osteomyelitis, and carried out a large number of studies to lay a better foundation for future development.

The network map of co-occurrence analysis of authors and cited literatures consisted of 131 nodes and 619 edges (Figure 5C). The results showed that the performance of Lipsky BA group and Zimmerli $W$ group were more remarkable (Figure 5C, Table 4).

\section{Analysis of the osteomyelitis hotspots}

The network map of co-occurrence analysis of keywords included 40 nodes and 186 edges (Figure 6). The top 5 keywords included osteomyelitis [2,698], infection [1,331], management [828], diagnosis [683], bone [552]. Centrality analysis showed that "therapy" had more impact than any other keywords (centrality $=0.21$ ), followed by disease [0.19], infection [0.17], stapbylococcus aureus [0.17], and management [0.16] (Table 5). The log-likelihood ratio (LLR) method was used for keywords clustering, and then keywords with
$\mathrm{OR} \geq 8$ and $\mathrm{P} \leq 0.005$ were selected. As shown in Table 6, the clustering results included five clusters, namely, spinal inflammation, prevention and treatment of infection, osteoarticular infection in children, diabetic foot and its complications, and fracture reconstruction. We finally analyzed the temporal trend of hotspot shift according to the top 25 terms with the strongest citation bursts during 2010 to 2019 (Table 7).

\section{Discussion}

Our study found that the research of osteomyelitis increased gradually in the 10 years from 2010 to 2019, and more and more scholars focused their attention on this field. Although the research has been very extensive, it is relatively messy and lack of analysis of research hotspots. In this work, we focused on the global state of research and trends in osteomyelitis so as to explore the research hotspots and frontiers. 
Table 3 The top 20 co-occurrence authors and co-cited authors contributed to publications in osteomyelitis research

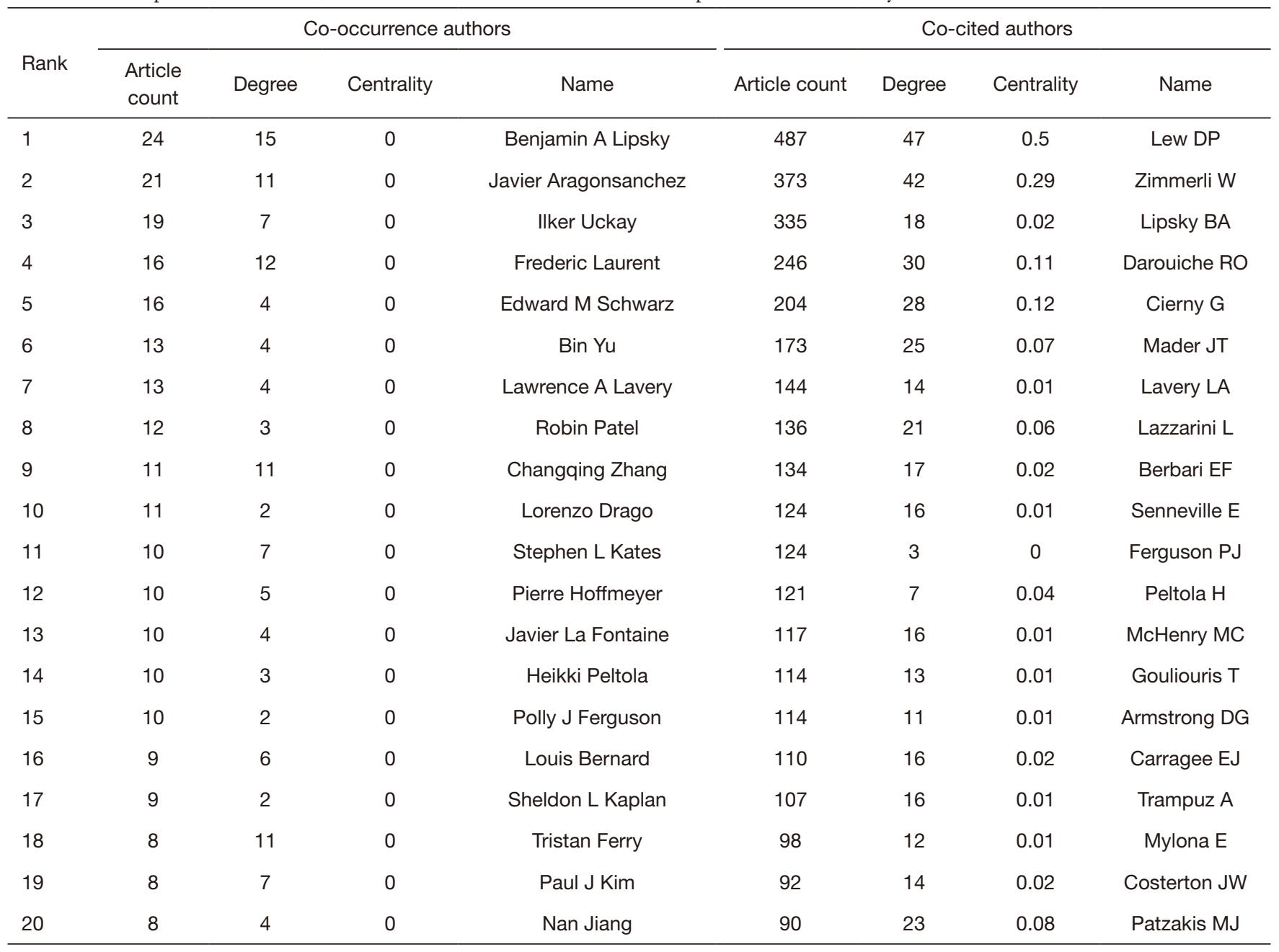

Table 4 The top 5 high-cited papers in osteomyelitis research during 2010 to 2019

\begin{tabular}{|c|c|c|c|c|c|}
\hline Rank & Total citations & Title & Journal & Author & Publication year \\
\hline 2 & 101 & $\begin{array}{l}\text { Spondylodiscitis: Update on Diagnosis and } \\
\text { Management }\end{array}$ & $\begin{array}{l}J \text { Antimicrob } \\
\text { Chemother }\end{array}$ & Gouliouris T & 2010 \\
\hline 4 & 94 & $\begin{array}{l}2015 \text { Infectious Diseases Society of America } \\
\text { (IDSA) Clinical Practice Guidelines for the } \\
\text { Diagnosis and Treatment of Native Vertebral } \\
\text { Osteomyelitis in Adults }\end{array}$ & Clin Infect Dis & Berbari EF & 2015 \\
\hline 5 & 92 & $\begin{array}{l}\text { Pyogenic Vertebral Osteomyelitis: A } \\
\text { Systematic Review of Clinical Characteristics }\end{array}$ & $\begin{array}{l}\text { Semin Arthritis } \\
\text { Rheum }\end{array}$ & Mylona E & 2009 \\
\hline
\end{tabular}




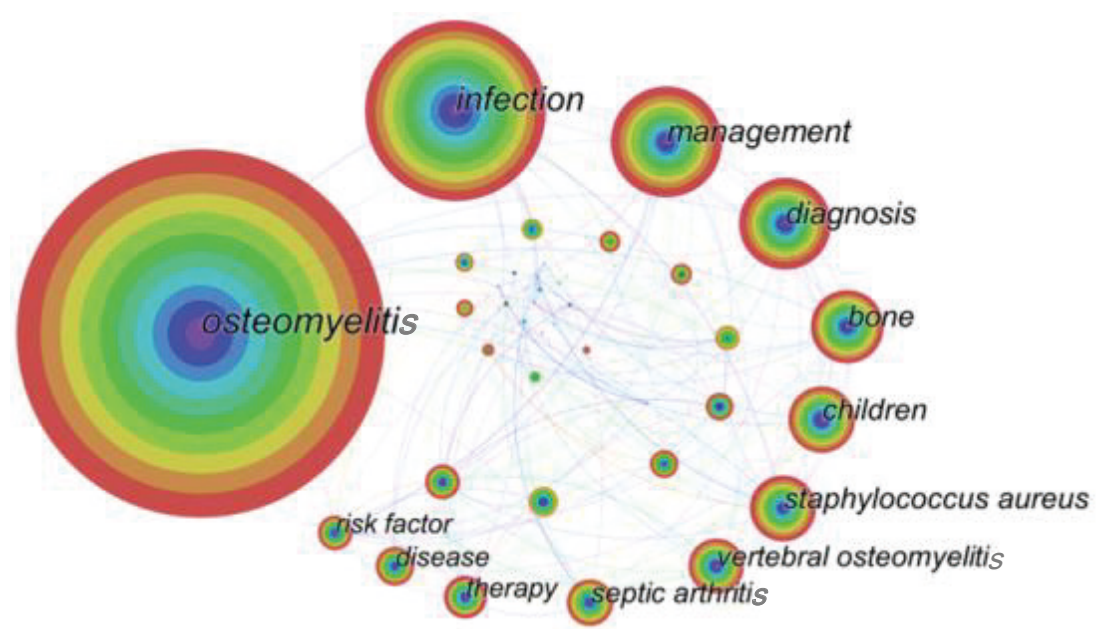

Figure 6 The network map of co-occurrence analysis of keywords. In the map, the nodes represent the analyzed objects, and the more frequency, the larger the nodes. The color and thickness in the inner circle of the node indicated the occurrence or cited frequency of different time periods. The edge between nodes and their thickness represents the relationship and strength of co-occurrence or co-citation, respectively. The nodes with centrality $\geq 0.1$ were marked with purple circle.

Table 5 The top 20 keywords in the field of osteomyelitis research

\begin{tabular}{|c|c|c|c|c|}
\hline Rank & Article count & Degree & Centrality & Keywords \\
\hline 2 & 1,331 & 17 & 0.17 & Infection \\
\hline 3 & 828 & 16 & 0.16 & Management \\
\hline 4 & 683 & 14 & 0.12 & Diagnosis \\
\hline 6 & 510 & 12 & 0.05 & Children \\
\hline 7 & 506 & 17 & 0.17 & Staphylococcus aureus \\
\hline 8 & 415 & 11 & 0.12 & Vertebral osteomyelitis \\
\hline 9 & 362 & 12 & 0.04 & Septic arthritis \\
\hline 12 & 271 & 14 & 0.11 & Risk factor \\
\hline 13 & 264 & 11 & 0.1 & Spondylodiscitis \\
\hline 14 & 233 & 9 & 0.08 & Complication \\
\hline 15 & 223 & 10 & 0.02 & Vancomycin \\
\hline 16 & 219 & 12 & 0.08 & Surgery \\
\hline 17 & 203 & 10 & 0.04 & In vitro \\
\hline 18 & 169 & 10 & 0.06 & Chronic osteomyelitis \\
\hline 19 & 167 & 10 & 0.01 & Antibiotics \\
\hline
\end{tabular}


Table 6 Cluster analysis results of keywords

\begin{tabular}{|c|c|c|c|}
\hline Cluster & Size & Silhouette & Keywords (log-likelihood ratio OR, P) \\
\hline 1 & 10 & 0.85 & $\begin{array}{l}\text { Staphylococcus aureus }(12.98,0.001) \text {; spondylodiscitis }(11.96,0.001) \text {; infection }(11.91,0.001) \text {; in vitro } \\
(11.53,0.001) \text {; release }(10.58,0.005) \text {; biofilm }(10.58,0.005) \text {; hydroxyapatite }(10.58,0.005) \text {; gentamicin } \\
(9.65,0.005) \text {; tuberculosis }(8.5,0.005)\end{array}$ \\
\hline 3 & 6 & 0.734 & $\begin{array}{l}\text { Diabetic foot }(30.68,0.0001) \text {; foot ulcer }(17.48,0.0001) \text {; tuberculosis }(9.19,0.005) \text {; peripheral arterial } \\
\text { disease }(8.72,0.005) \text {; diabetic foot infection }(8.72,0.005) \text {; mandible }(8.72,0.005) \text {; diabetic foot } \\
\text { infections }(8.72,0.005) \text {; revascularization }(8.72,0.005)\end{array}$ \\
\hline
\end{tabular}

Regarding the contributions of countries/regions and institutions, developed countries in Europe and the United States occupied the main position in the field of global osteomyelitis research. The United States, Germany, England, and France were all in the top 5 countries in terms of number of articles published and centrality. The United States seems to have superior conditions for basic and clinical medical research, which include sufficient funds, advanced equipment, and professional researchers. Among the outstanding institutions, Mayo Clinic, University of Washington, University of California, San Francisco, Harvard University, Duke University, Baylor Medical School and Vanderbilt University are all from the United States. Surprisingly, although China ranked second in the number of publications, its centrality was not optimistic. However, the performance of two institutions from China (Shanghai Jiao Tong University, and Chang Gung University) were commendable. Generally, the strength of scientific research from China needs to be further improved. If there is sufficient communication and cooperation between institutions in various countries, the research on osteomyelitis will make an enormous breakthrough.

Regarding the distribution of journals publishing researches on osteomyelitis, the fournal of Foot Ankle Surgery published 111 studies in this area, far ahead of other journals. The journal mainly covers the research progress in the field of foot and ankle surgery and may pay more attention to the related research of diabetic foot. Other journals, including Pediatric Infectious Disease Fournal, Injury International Fournal of the Care of the Injured, PLoS
One and BMC Infectious Diseases, were the primary journals containing osteomyelitis publications. It is worth noting that the journal Clin Infect Dis, which has published many clinical practice guidelines related to bone infection in the past, have been cited 2,275 times and was much higher than that of other top orthopedic journals, such as The fournal of Bone and foint Surgery-American Volume, Clinical Orthopaedics and Related Research, and The Fournal of Bone and Foint Surgery-British Volume. In addition, the articles published by the famous international top journal New England Fournal of Medicine and Lancet are of high quality, and they are also in the forefront in the frequency of citation. Therefore, these findings indicate that future developments in the field may be published in the above-mentioned journals.

Notably, a number of research groups of collaborators are being formed in the world. Among them, Lew DP, Zimmerli W, Lipsky BA, Darouiche RO and other researchers are the most representative and influential scholars in the field of osteomyelitis research in the world. Professor Lew DP has comprehensively and carefully summarized the etiology, pathogenesis, diagnosis and treatment of osteomyelitis, which is of far-reaching significance to guide the followup clinical and basic research of osteomyelitis (1). Dr. Lipsky has been committed to the research related to the diagnosis and treatment of diabetic foot for a long time, and has participated in the formulation of a number of clinical practice guidelines (13-15). His group not only published the largest numbers of papers in this field but also published their own highly cited representative papers in top journals. The team of Dr. Professor Zimmerli W, an internationally 
Table 7 The top 25 keywords with the strongest citation bursts during 2010 to 2019

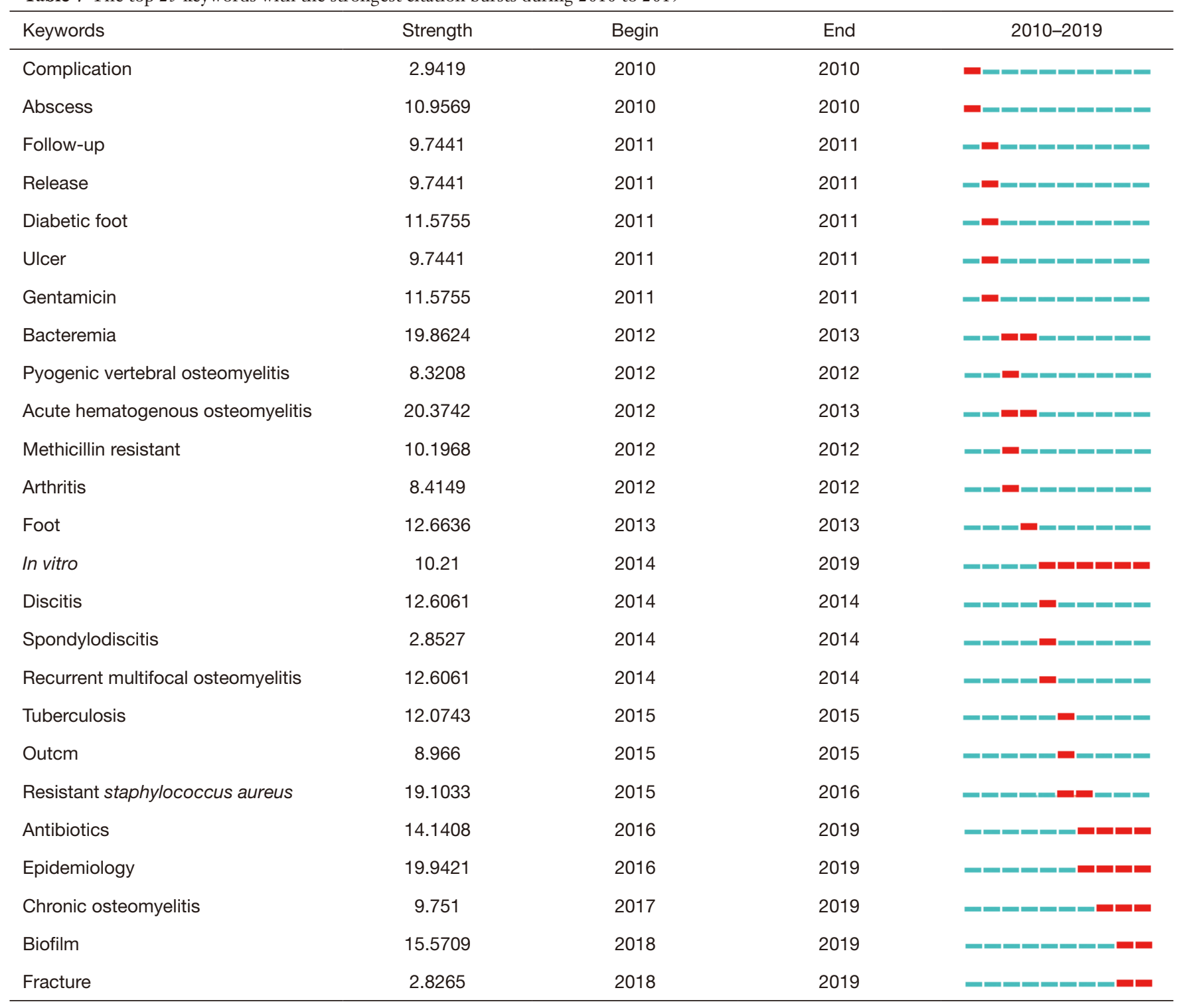

The color red means that there was an outbreak of the keyword in this year.

renowned expert in the field of bone and joint infection, and masters such as Metsemakers WJ, Morgenstern M, McNally MA and Trampuz A are members of the consensus group on Fracture-Related Infection. Several scholars have also made tremendous achievements in osteomyelitis research, consisting of Javier Aragón-Sánchez, Ilker Uçkay, Edward M. Schwarz, and Frederic Laurent. Additionally, some scholars from China are also gradually emerging, such as Bin Yu, Changqing Zhang, Zhao Xie and so on. They are now active in the frontline of bone infection research. Obviously, our study demonstrates that these scholars have played an influential core role in the osteomyelitis field and carried out substantial research to lay a solid foundation for future development.

From the analysis of keywords, we can see that the research on the management, diagnosis, and treatment of Staphylococcus aureus infection and osteomyelitis has attracted much attention in the past 10 years. The keywords with the strongest citation bursts can reflect the development trend and mutation points of a certain discipline to a certain extent, and reveal the research direction with potential value. We found that the focus of the researchers was 
different at different stages of the study. In the early stage (from 2010 to 2013), researchers paid more attention to the clinical manifestations of bone and joint infectious diseases and the management of their complications, such as diabetic foot, acute hematogenous osteomyelitis, septic arthritis, abscesses, ulcers, bacteremia, etc. As time progresses, in vitro research has received continuous attention. In the later stage (from 2014 to 2019), the epidemiology and treatment of osteomyelitis (such as surgery, antibiotics, biofilm, outcome) have become much more important research hotspots. There is no doubt that the research on the diagnosis, treatment and management strategy of osteomyelitis have always received extensive attention from researchers.

So far, there are still many confusions and controversies in the diagnosis and treatment of osteomyelitis. The care of patients with osteomyelitis is multidisciplinary, requiring the communication and collaboration among orthopedics, radiology, microbiology, infectious diseases, nursing and community teams to obtain early diagnosis and effective treatment. In order to address this issue, an international expert group on fracture-related infection comprised of a number of scientific and medical organizations has been convened, with the support of the AO Foundation (16). They have conducted comprehensive and in-depth research on fracture-related infections, including its definition, pathogenesis and management, preclinical in vivo models, treatment and outcome, current surgical and microbiological concepts, prevention, diagnostic challenges and future perspectives (16-22).

Nonetheless, there will inevitably be some limitations in this study. The database is constantly updated, and we have only selected the literature from 2010 to 2019, without those literatures published after that day. Moreover, the literature we analyzed contains only English literature, not those in other languages, so there will be some bias. Therefore, there will be a discrepancy between our bibliometrics analysis and the actual publishing situation. With the widespread attention of researchers and the progress of technology, the future research on osteomyelitis may show an explosive growth.

\section{Conclusions}

In this study, we summarized the publication information of osteomyelitis-related literature in the 10 years from 2010 to 2019, including countries/regions and institutions, publication journals, authors, and keywords. We then analysed the research hotspots in the osteomyelitis field based on these studies. There is no doubt that the research on the diagnosis, treatment and management strategy of osteomyelitis have attracted extensive attention from researchers. The research on the pathogenesis and treatment methods of osteomyelitis will be the trend and hotspots in the future. In conclusion, we believe our research can help researchers to understand the current research status and hotspots of osteomyelitis from a macroscopic view, which can make it easier for achieving major scientific breakthroughs someday.

\section{Acknowledgments}

Funding: This study was funded by the Health Scientific Innovation Platform Program of Fuzhou (2019-Swp3), Natural Science Foundation of Fujian Province (2020J011186) and Fuzhou Science and Technology Projects (2020-WS-87).

\section{Footnote}

Data Sharing Statement: Available at http://dx.doi. org/10.21037/apm-20-1978

Peer Review File: Available at http://dx.doi.org/10.21037/ apm-20-1978

Conflicts of Interest: All authors have completed the ICMJE uniform disclosure form (available at http://dx.doi. org/10.21037/apm-20-1978). The authors have no conflicts of interest to declare.

Ethical Statement: The authors are accountable for all aspects of the work in ensuring that questions related to the accuracy or integrity of any part of the work are appropriately investigated and resolved.

Open Access Statement: This is an Open Access article distributed in accordance with the Creative Commons Attribution-NonCommercial-NoDerivs 4.0 International License (CC BY-NC-ND 4.0), which permits the noncommercial replication and distribution of the article with the strict proviso that no changes or edits are made and the original work is properly cited (including links to both the formal publication through the relevant DOI and the license). 
See: https://creativecommons.org/licenses/by-nc-nd/4.0/.

\section{References}

1. Lew DP, Waldvogel FA. Osteomyelitis. Lancet 2004;364:369-79.

2. Gutierrez K. Bone and joint infections in children. Pediatr Clin North Am 2005;52:779-94.

3. Yeo A, Ramachandran M. Acute haematogenous osteomyelitis in children. BMJ 2014;348:g66.

4. Kavanagh N, Ryan EJ, Widaa A, et al. Staphylococcal Osteomyelitis: Disease Progression, Treatment Challenges, and Future Directions. Clin Microbiol Rev 2018;31:e00084-17.

5. Chen C. Searching for intellectual turning points: progressive knowledge domain visualization. Proc Natl Acad Sci U S A 2004;101 Suppl 1:5303-10.

6. Chen C, Song M. Visualizing a field of research: A methodology of systematic scientometric reviews. PLoS One 2019;14:e223994.

7. Synnestvedt MB, Chen C, Holmes JH. CiteSpace II: visualization and knowledge discovery in bibliographic databases. AMIA Annu Symp Proc 2005;2005:724-8.

8. Chen $\mathrm{C}, \mathrm{Hu} \mathrm{Z}$, Liu S, et al. Emerging trends in regenerative medicine: a scientometric analysis in CiteSpace. Expert Opin Biol Ther 2012;12:593-608.

9. Guo S, Wang L, Xie Y, et al. Bibliometric and Visualized Analysis of Stem Cells Therapy for Spinal Cord Injury Based on Web of Science and CiteSpace in the Last 20 Years. World Neurosurg 2019;132:e246-58.

10. Wang SQ, Gao YQ, Zhang C, et al. A Bibliometric Analysis Using CiteSpace of Publications from 1999 to 2018 on Patient Rehabilitation After Total Knee Arthroplasty. Med Sci Monit 2020;26:e920795.

11. Tao L, Zhou S, Tao Z, et al. The publication trends and hot spots of scoliosis research from 2009 to 2018: a 10year bibliometric analysis. Ann Transl Med 2020;8:365.

Cite this article as: Chen $\mathrm{P}$, Lin $\mathrm{X}$, Chen $\mathrm{B}$, Zheng $\mathrm{K}$, Lin $\mathrm{C}, \mathrm{Yu} \mathrm{B}$, Lin $\mathrm{F}$. The global state of research and trends in osteomyelitis from 2010 to 2019: a 10-year bibliometric analysis. Ann Palliat Med 2021;10(4):3726-3738. doi: 10.21037/apm20-1978
12. Landis JR, Koch GG. The measurement of observer agreement for categorical data. Biometrics 1977;33:159-74.

13. Lipsky BA, Berendt AR, Cornia PB, et al. 2012 Infectious Diseases Society of America clinical practice guideline for the diagnosis and treatment of diabetic foot infections. Clin Infect Dis 2012;54:e132-73.

14. Lipsky BA, Aragon-Sanchez J, Diggle M, et al. IWGDF guidance on the diagnosis and management of foot infections in persons with diabetes. Diabetes Metab Res Rev 2016;32 Suppl 1:45-74.

15. Wukich DK, Armstrong DG, Attinger CE, et al. Inpatient management of diabetic foot disorders: a clinical guide. Diabetes Care 2013;36:2862-71.

16. Metsemakers WJ, Morgenstern M, McNally MA, et al. Fracture-related infection: A consensus on definition from an international expert group. Injury 2018;49:505-10.

17. Depypere M, Morgenstern M, Kuehl R, et al. Pathogenesis and management of fracture-related infection. Clin Microbiol Infect 2020;26:572-8.

18. Vanvelk N, Morgenstern M, Moriarty TF, et al. Preclinical in vivo models of fracture-related infection: a systematic review and critical appraisal. Eur Cell Mater 2018;36:184-99.

19. Bezstarosti H, Van Lieshout E, Voskamp LW, et al. Insights into treatment and outcome of fracture-related infection: a systematic literature review. Arch Orthop Trauma Surg 2019;139:61-72.

20. Metsemakers WJ, Kuehl R, Moriarty TF, et al. Infection after fracture fixation: Current surgical and microbiological concepts. Injury 2018;49:511-22.

21. Metsemakers WJ, Onsea J, Neutjens E, et al. Prevention of fracture-related infection: a multidisciplinary care package. Int Orthop 2017;41:2457-69.

22. Morgenstern M, Kuhl R, Eckardt H, et al. Diagnostic challenges and future perspectives in fracture-related infection. Injury 2018;49 Suppl 1:S83-90. 